\title{
New Propellant Component, Part II. Study of a PSAN/ DNAM/HTPB Based Formulation
}

\author{
P. Simões*, L. Pedroso, and A. Portugal \\ LEDAP_Laboratory of Energetics and Detonics, Chemical Engineering Department, Faculty of Sciences \\ and Technology, University of Coimbra, 3030-290 Coimbra (Portugal)
}

\section{Plaksin and J. Campos}

LEDAP_Laboratory of Energetics and Detonics, Mechanical Engineering Department, Faculty of Sciences and Technology, University of Coimbra, 3030-290 Coimbra (Portugal)

\section{Summary}

A study of DNAM as a candidate ingredient for propellant formulations is reported. A formulation including DNAM and based on Phase Stabilized Ammonium Nitrate (PSAN) and Hydroxy-Terminated-PolyButadiene (HTPB) was selected for the study. This includes thermoanalytical measurements on the mixtures of solid components and propellant samples. Performance is assessed by burning rate measurements. A new small-scale shock sensitivity test developed for studying the propellant under consideration is described. A good potential for DNAM was found for this formulation as revealed by the performance and low vulnerability of the PSAN/DNAM/HTPB composition.

\section{Introduction}

The research and development of new energetic materials must follow a systematic approach. A suitable scheme, involving different but logically inter-related stages, should be implemented from the initial synthesis to the inclusion in a final energetic device. Characterization is an essential issue remaining as such along the overall development process $^{(1)}$. This means that there is a moment in which studies on the ingredients themselves, and those subsequently directed to their incorporation in suitable formulations, should be conducted side by side to allow the redefinition and/or optimization procedures in the light of the accumulated knowledge.

Like other well-known nitramines, DNAM is not a true oxidizer in the sense that it has an oxygen deficiency, as exhibited by its oxygen balance $-18.42 \%$. This means that a suitable propellant formulation including DNAM as a solid load ingredient should also include either an adequate energetic binder system or, if an inert is chosen, an energetic filler with a positive oxygen balance. Hydroxyl-terminatedpolybutadiene (HTPB), known as a state-of-art hydrocarbon binder, was selected in our study. In order to compensate its

\footnotetext{
* Corresponding author; e-mail: pnsim@eq.uc.pt
}

low oxygen content, the incorporation of an oxygen rich component was necessary, and ammonium nitrate (AN) was selected as a conventional oxidizer. The use of AN in explosive and propellant formulations is well known. Low cost, chemical stability, low sensitivity to friction and to shock, as well as its low-signature/polluting characteristics are strong reasons for the selection. Its use as a main propellant ingredient has been limited by its high hygroscopicity, solid state phase transition characteristics, particle shape, low energy and low reactivity. Nevertheless, there has been a renewed interest on $\mathrm{AN}$ in the last years ${ }^{(2-7)}$. To reduce its low performance characteristics, AN has tentatively been formulated with metals ${ }^{(5)}$, as well as with energetic binders ${ }^{(2,3)}$. Furthermore, a lot of effort has been put in developing different kinds of phase stabilized ammonium nitrate (PSAN) ${ }^{(8,9)}$ to minimize the undesirable physical properties and its effects.

This work reports the assessment of DNAM potential as a propellant ingredient in a PSAN and HTPB based formulation. Thermoanalytical studies have been carried out on the mixtures of solid ingredients and propellant samples. Performance has been evaluated by measuring and comparing regression rates of $\mathrm{PSAN} / \mathrm{HTPB}$ and PSAN/DNAM/ HTPB based formulations. A new small-scale test has been developed to study the shock sensitivity of propellant samples. The work developed up to now allowed us to clarify the main DNAM attributes and therefore, to open new avenues for its application.

\section{Studies on a PSAN/DNAM/HTPB Based Propellant}

The PSAN used in this study (from ICT, Germany) included $1.0 \% \mathrm{NiO}$, as phase stabilizing agent, and $0.5 \%$ Petro (sodiumlaurylsulfonate), as anti-caking agent.

Two propellant compositions were prepared for testing (percentages in mass): 
- PC1: PSAN (80\%), HTPB (20\%);

- PC2: PSAN (68\%), DNAM (12\%), HTPB (20\%).

After mixing the components, isophorone-diisocyanate (IPDI) was used as curing agent. The curing process was carried out at $40^{\circ} \mathrm{C}$ for 10 days. The density of propellant samples was measured by helium picnometry (MicrometricsAccuPyc1330) and values higher than $99 \%$ of the theoretical maximum density were found.

Preliminary results based on vacuum stability tests indicated that compatibility problems between DNAM and HTPB should not be excluded. The acidity of primary nitramine groups in DNAM may play an important role in a possible reactive interaction with HTPB. However, our present knowledge on the chemistry of DNAM does not permit the postulating of any detailed mechanism.

\subsection{Thermoanalytical Measurements}

\subsubsection{Thermal Behavior of Solid Mixtures of DNAM and PSAN}

The thermal decomposition of AN has been widely studied $^{(10-17)}$, but a considerably lower number of works on the thermal behavior of PSAN has been reported. A recent study on this subject can be found in Ref. (18).

The thermal behavior of solid mixtures of DNAM and PSAN over the DNAM/PSAN mass ratios ranging from $10 / 90$ to $90 / 10$ was recently investigated ${ }^{(19)}$. The results are summarized in Figure 1 in the form of thermoanalytical curves (the thermoanalytical results reported in this paper were performed on a Rheometric Scientific - STA 1500 equipment, at $10^{\circ} \mathrm{C} \mathrm{min}^{-1}$, using $\mathrm{N}_{2}$ as purge gas).

It was found that the decomposition starts when both components are in the solid state. Thermomicroscopy was used and the visual inspection of the process was correlated with detailed thermoanalytical results. This clearly indicated that DNAM starts to decompose slowly at about $123-124^{\circ} \mathrm{C}$.

If the substantial lowering of the decomposition temperature of DNAM in the mixtures can be considered as a potential problem from the point of view of compatibility between components, it can also be interpreted as a favorable energetic and reactive enhancement in the overall decomposition process. In fact, as the DNAM content in the mixture increases, the endothermic effects due to the melting and decomposition of PSAN tend to disappear. PSAN is the component whose thermal behavior limits the exothermic character of the overall decomposition process, but it also plays an important role in the decomposition of DNAM. The way how this reaction proceeds once initiated is rather conditioned by the liquid mass resulting from the melting of PSAN formed after the II $\rightarrow$ I transition. Thermomicroscopy visualizes that the first decomposition stage may be regarded as a synergetic coexistence of the exothermic decomposition of DNAM and the melting of PSAN, particularly for compositions up to $60 \%$ in DNAM. The beginning of the decomposition in the solid state contributes as an extra heat source for melting PSAN, which, in turn, provides a

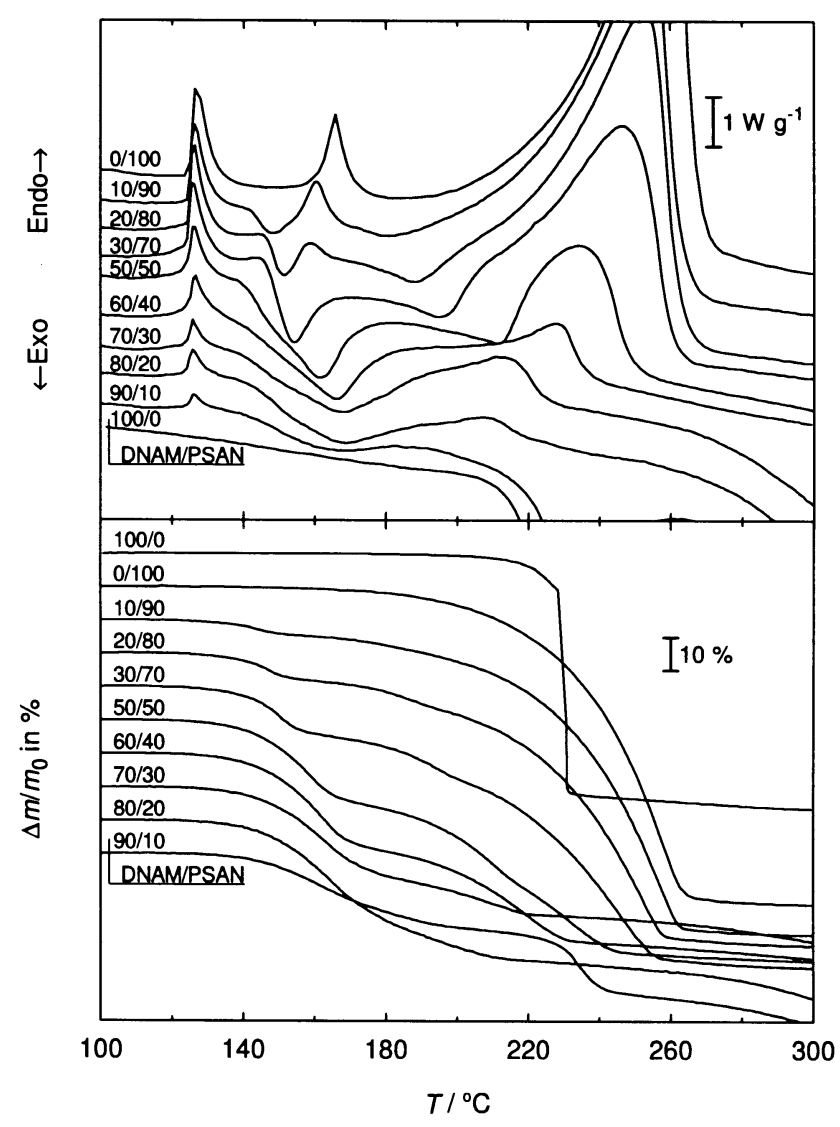

Figure 1. Thermoanalytical curves of DNAM/PSAN solid mixtures at $10^{\circ} \mathrm{C} \mathrm{min}{ }^{-1}$.

liquid medium that accelerates the decomposition of DNAM. As the amount of PSAN is decreased in the mixtures, the corresponding lower liquid phase leads to remarkable changes in the overall decomposition process. For these cases, an early exothermic peak ascribable to the thermolysis of DNAM is still being detected, although in a more limited extent, indicating that only a partial and slower decomposition occurs at lower temperatures ${ }^{(19)}$.

\subsubsection{Thermal Behavior of Propellant Samples}

The results of this thermoanalytical study are presented in Figure 2. A shift of the curves towards lower temperatures is globally observed for the composition PC2 when compared with $\mathrm{PC} 1$. The endothermic peak temperatures due to the phase transitions IV $\rightarrow$ II and II $\rightarrow$ I are shifted by less than $2{ }^{\circ} \mathrm{C}$, while the melting peak appears shifted by about $5{ }^{\circ} \mathrm{C}$. The largest difference occurs for the main exothermic peak, being $24{ }^{\circ} \mathrm{C}$ between the two cases, and somewhat more energetic for PC2. The first detectable mass loss (which indicates the onset of some reactive event) occurs in the approximate range of $142-145^{\circ} \mathrm{C}$ for $\mathrm{PC} 2$, which is about $10-13{ }^{\circ} \mathrm{C}$ before the start of the decomposition of $\mathrm{PC} 1$. Thus, the magnitude of this shift is considerably lower than those corresponding to the start of the onset of the decomposition in DNAM/PSAN mixtures compared to the onset of each component alone (compare Figures 1 and 2). This suggests 


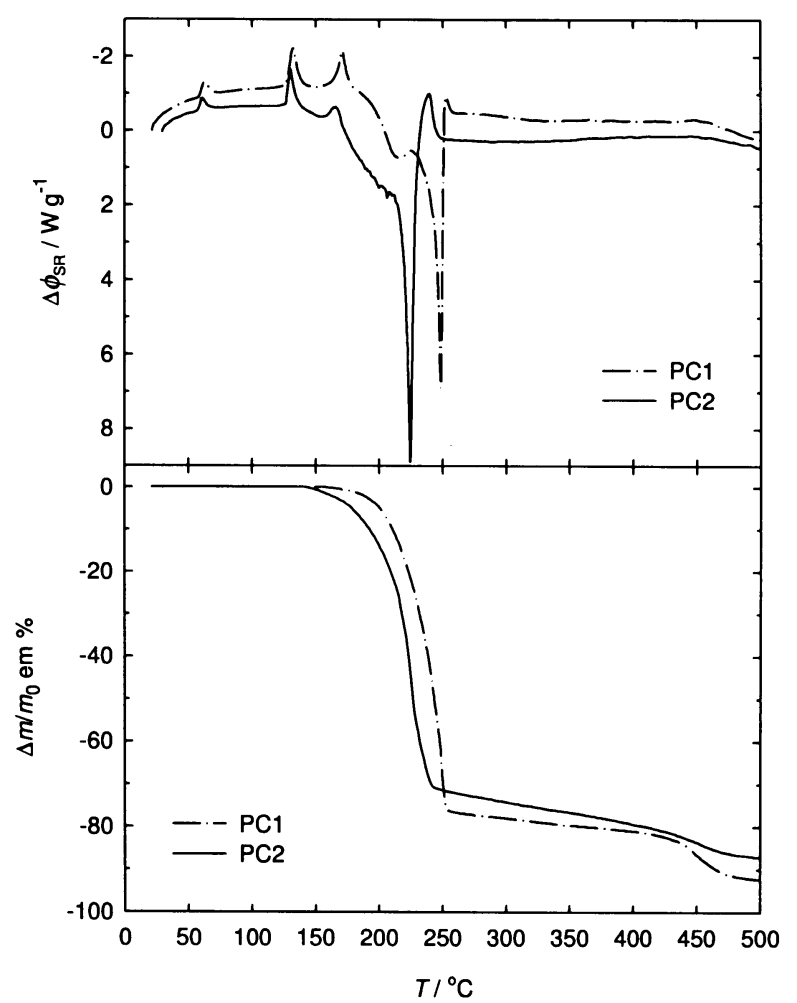

Figure 2. Thermoanalytical curves of propellants $\mathrm{PC} 1$ and $\mathrm{PC} 2$ at $10{ }^{\circ} \mathrm{C} \min ^{-1}$.

that the binder limits the interaction between DNAM and PSAN which was observed from mixtures of these components in a comparable temperature range. However, as it can be observed in the detailed view of Figure 2, a noticeable increase in the mass loss rate within the approximate temperature range of $220-230^{\circ} \mathrm{C}$ occurs. This effect can be attributed to the decomposition of DNAM which - taking into account the temperature range of its occurrence - should coexist with the decomposition of melted PSAN. Since this occurs in the propellant by means of an exothermic reaction, a global energetic and kinetic enhancement is achieved.

\subsubsection{Regression Rate Measurements}

The combustion regression rate was assessed to compare the performance of the two propellant compositions. The experimental measurements were performed on a strand burner in $\mathrm{N}_{2}$ atmosphere, within a pressure range of 2-10 MPa and at ambient temperature. The experimental points and correspondent fitting lines are plotted in Figure 3, and the fitting results for the Vieille's law model, $r=a \cdot p^{n}$, are summarized in Table 1.

These results show that an increase in the regression rate of $13 \%$ is obtained when PSAN is replaced by DNAM in an amount of $12 \%$. Furthermore, in the pressure range studied, the same value for the pressure exponent, $n$, is obtained. These results substantiate the good burning characteristics of DNAM and its ability to improve the combustion behavior of PSAN/HTPB based propellants, and hopefully other propellant compositions.

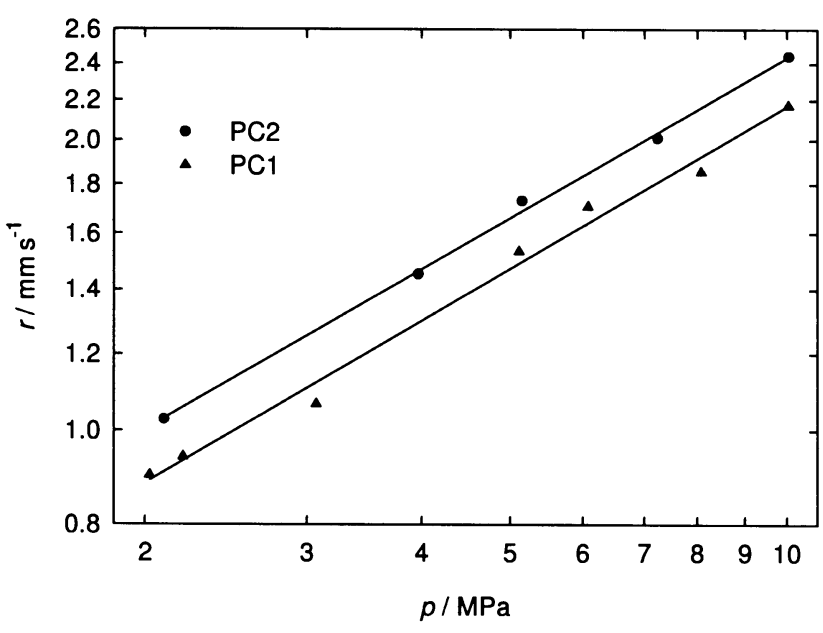

Figure 3. Results of the regression rate measurements of propellants $\mathrm{PC} 1$ and PC2.

Table 1. Fitting Results of the Model $r=a \cdot p^{n}$

\begin{tabular}{lccc}
\hline Propellant & $a$ & $n$ & $\gamma^{*}$ \\
\hline PC1 & 0.61 & 0.55 & 0.9957 \\
PC2 & 0.69 & 0.55 & 0.9990 \\
\hline
\end{tabular}

* Correlation coefficient.

\subsubsection{Shock Sensitivity Measurements}

A new experimental setup, that appears to become a promising technique, has been developed for studying the shock sensitivity of the PC2 propellant. The setup included an explosive plane wave generator (PWG) together with an original optical registration method as a diagnostic source to determine the shock propagation through the sample. The shock wave propagation process is registered in a quasicontinuous way by using a thin multi-fibre optical strip, consisting in 89-98 fibres of $250 \mu \mathrm{m}$ diameter, i.e., $89-98$ independent channels connected directly to a fast electronic streak camera (Thomson TSN 506 N, see Figure 4(a)). A schematic illustration of the PWG is shown in Figure 4(b). It consisted in a PVC confined cylindrical plastic explosive charge, together with a variable number of PMMA slabs. The flatness of the shock front produced by the PWG was checked before the experiments with the propellant samples were performed. The result of this test is shown in the photo-chronogram of Figure 4(b), where it is possible to see that the slight curvature in the shock front is approximately constant as it propagates within 15 to $25 \mathrm{~mm}$ in the PMMA barrier.

Propellant samples with a cylindrical shape $(30 \mathrm{~mm}$ diameter and $15 \mathrm{~mm}$ thickness) were cut in a predetermined angle from the longitudinal axis for inserting on the optical device (fibre strip plus shock-light converter; see below), and then glued together as shown in Figure 5(a). The photoregistration of the shock front is made through the side surface of the multi-fibre strip. For this purpose, the gaps 


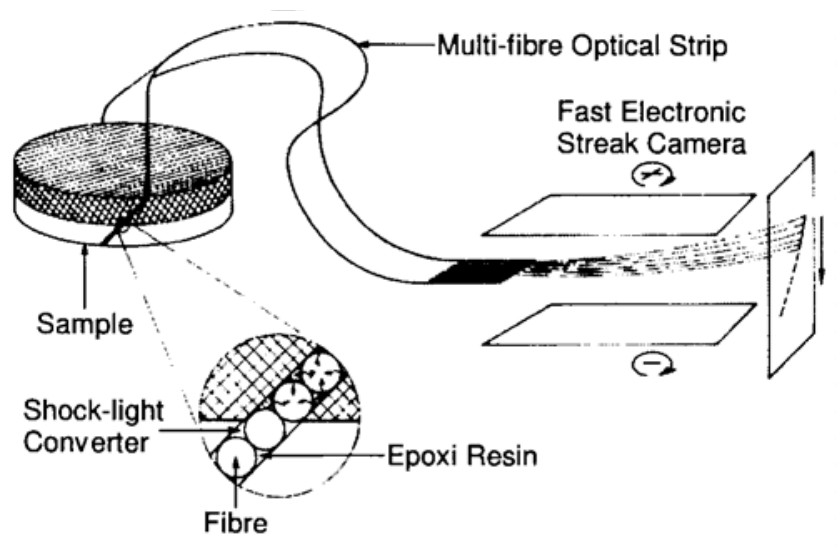

(a) Optical registration method.

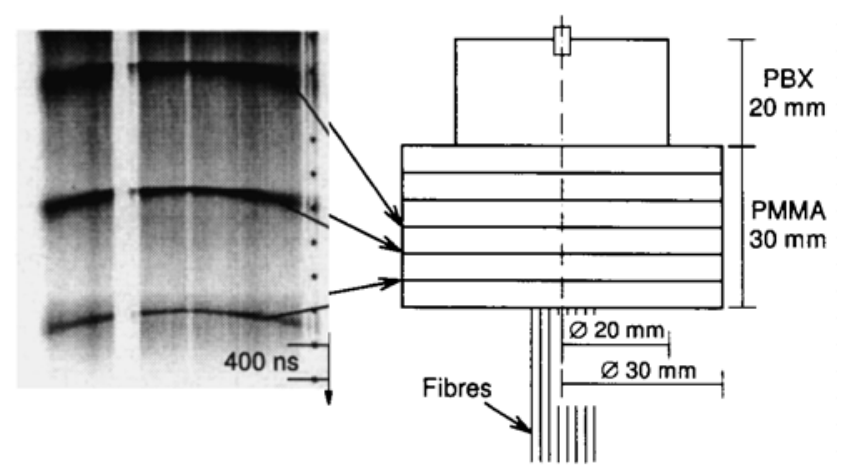

(b) PWG arrangement and photo-chronogram for checking shock wave flatness.

Figure 4. Basic components of the setup for studying the shock sensitivity.

between adjacent fibres and the surface of the shock loaded sample were filled by a shock-light converter (a material based on rhodamine) for the light irradiation by triboluminescense. The gaps of the opposite side of the multi-fibre strip, between the fibres and the second part of the sample, were filled by an epoxy resin. The light impulse is transmitted to the fibre through its side surface filled with the shock-light converter (see Figure 4(a)). The developed method allowed the registration, in the same experiment, of $x-t$ diagrams of the induced shock wave both in the PMMA barrier and in the sample. Temporal and spatial resolutions of $2 \mathrm{~ns}$ and $250 \mu \mathrm{m}$, respectively, are allowed by the applied optical method.

Four tests have been performed on PC2 propellant samples (e.g., see Figure 5(a)). The shock amplitudes in the PMMA barrier were ca. 1.3, 3.3, 7.6 and 9.0 GPa. Figure 5(b) presents a typical photo-chronogram. The $x-t$ diagrams and the shock velocities were obtained from the photo-registration (taking into account the angle according to which the strip was inserted into the sample), by evaluating the way it changed with the propagation distance in the sample as shown in Figure 5(c).

The Hugoniot curve of propellant PC2 has been calculated according to the impedance matching $\operatorname{method}^{(20)}$, and the

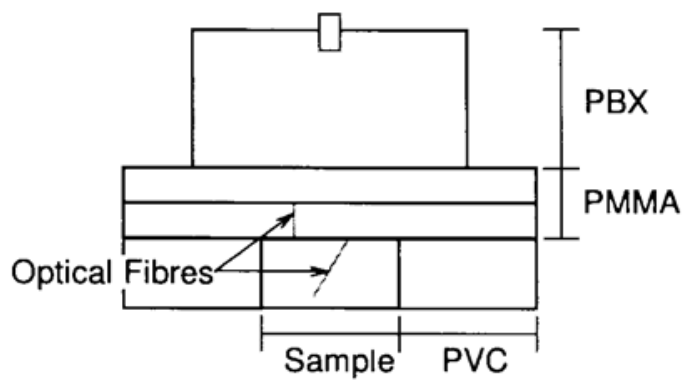

(a) PWG and propellant sample.

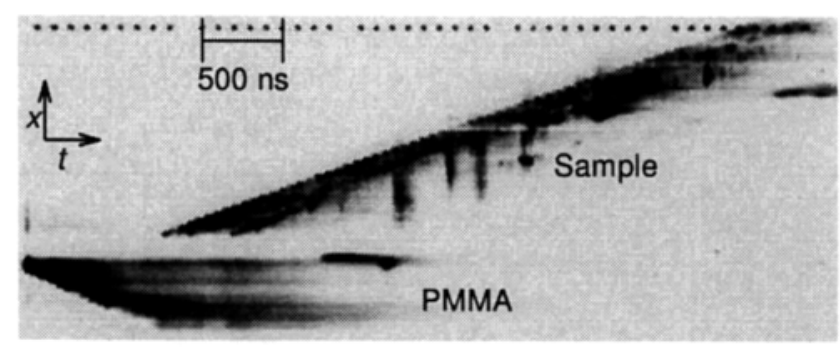

(b) Photo-chronogram.

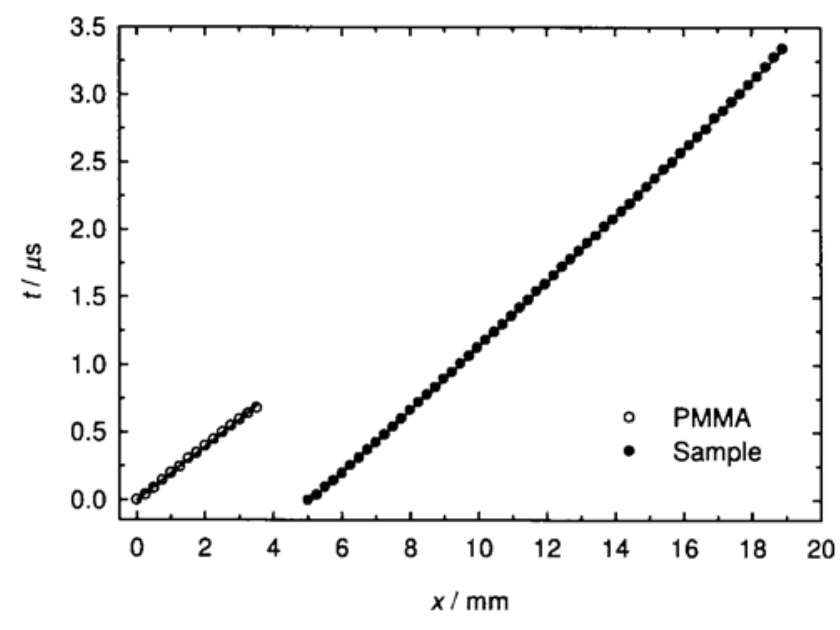

(c) $x-t$ diagram.

Figure 5. Typical experimental setup and test results for the propellant PC2.

results are resumed in Figure 6 by plotting the pressure, $p$, against the particle velocity, $U_{P}$. The $U_{S}$ values concerning both the PMMA and the sample were estimated taking the derivative of a fitted second order polynomial to the points as those presented in the $x-t$ diagram of Figure 5(c). Furthermore, the results were checked by comparing the $p$ and $U_{S}$ levels of each test of the sample with those obtained from the PWG alone. This has to be emphasized because this criterion was found to be the best way to get around some pitfalls in determining $U_{S}$. In fact, the $x-t$ data points present a slight curvature which - if not taken into account-leads to an inaccurate determination of $U_{S}$ and consequently to doubtful $p-U_{P}$ pairs. 


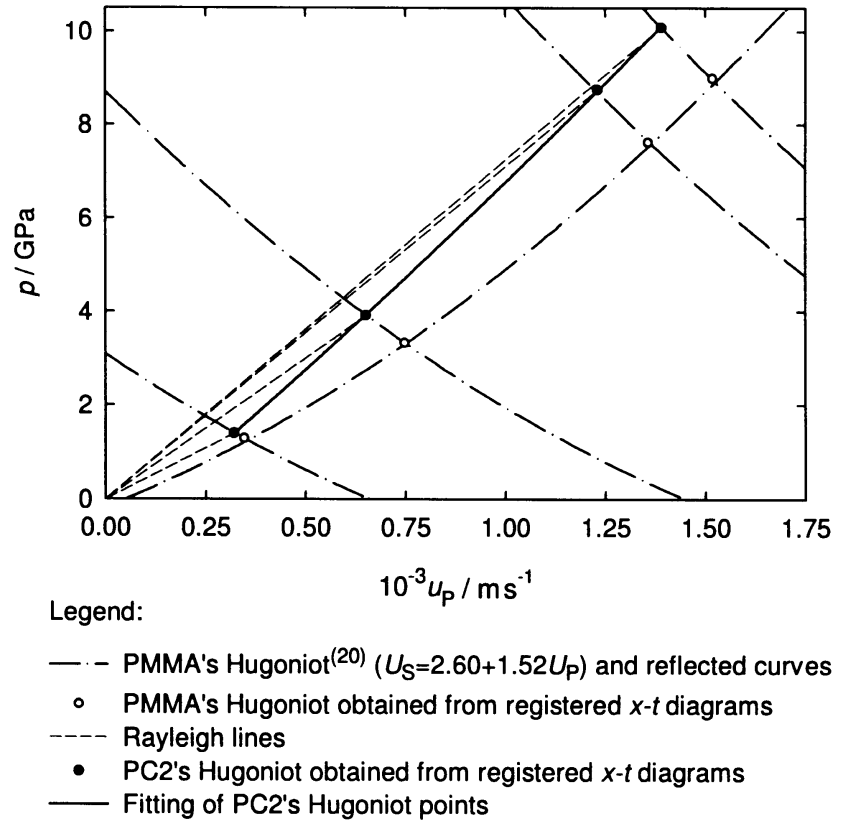

Figure 6. Evaluation of $\mathrm{PC} 2$ 's Hugoniot points.

This study revealed that no shock to detonation transition occurs in the propellant PC2 for a shock load up to about $9 \mathrm{GPa}$. This is in accordance with which could be anticipated from the low sensitivity of DNAM as a neat solid material, and clearly suggests that DNAM in this formulation has no problematic effects on the vulnerability of the propellant.

It should be mentioned that the applied method allows not only the registration of the first shock front (which has been analyzed in the present paper), but also the recording of the rear release wave as well as the intermediate shocks which can be related with possible phase transitions and/or shock induced chemical reactions. Analysis of the shock wave structure and its change in the process of shock wave propagation in propellants will be subject in our future studies.

\section{Conclusions}

The thermoanalytical investigations have shown that the thermal behavior of DNAM/PSAN is rather dependent on the composition. A chemical interaction between the components causes a shift towards lower temperatures on the onset of the decomposition of DNAM. A thermal coupling seems to occur between the thermolysis of DNAM and the melting of PSAN, mainly for compositions up to about $50 \%$ in DNAM. The beginning of the exothermic decomposition of DNAM contributes as an extra heat source for the melting of PSAN, which, in turn, provides a liquid medium supporting the growing of the DNAM decomposition. The global result is an enhancement in the reactivity of the mixture, which was confirmed by the thermoanalytical measurements of propellant samples including DNAM. The improvement in the burning characteristics has been clearly evidenced by the increase of the burning rate due to DNAM.

The shock sensitivity studies show that DNAM does not impair the low vulnerability requirements of propellants. Emphasis should also be given to the original framework developed for studying the shock sensitivity of propellants. In fact, only small amounts of samples are necessary, and the enhanced resolution allows a better insight in the shock wave structure.

\section{References}

(1) A. Sanderson, "The Characterisation and Development of New Energetic Materials", 27th Int. Annual Conference of ICT, Karlsruhe, Germany, June 25-28, 1996, pp. 18.1-18.8.

(2) Y. Oyumi, E. Kimura, S. Hayakawa, and K. Kato, "Insensitive Munitions (IM) and Combustion Characteristics of GAP/AN Composite Propellants", Propellants, Explosives, Pyrotechnics 21, 271-275, 1996.

(3) K. Menke, J. Böhnlein-Mauß, and H. Schubert, "Characteristic Properties of AN/GAP Propellants", Propellants, Explosives and Pyrotechnics 21, 139-145, 1996.

(4) D. Campbell, A. S. Cumming, and E. J. Marshall, "Development of Insensitive Propellants based on Ammonium Nitrate and PolyNIMMO", in: "Environmental Aspects of Rocket and Gun Propulsion" AGARD Conference Proceedings 559, Neuilly sur Seine 1995 , pp. 14.1-14.9.

(5) P. Carvalheira, G. M. H. J. L. Gadiot, and W. P. C. Klerk, "Thermal Decomposition of Phasestabilised Ammonium Nitrate (PSAN), Hydroxyl-Terminated Polybutadiene (HTPB) Based Propellants. The Effect of Iron (III) Oxide Burning-Rate Catalyst", Thermochimica Acta 269/270, 273-293, 1995.

(6) M. L. Chan and S. DeMay, "Development of Environmentally Acceptable Propellants", in: "Environmental Aspects of Rocket and Gun Propulsion" AGARD Conference Proceedings 559, Neuilly sur Seine 1995, pp. 9.1-9.5.

(7) R. A. Stowe, P. Lessard, G. Roy, and G. Richer, "Evaluation of Ammonium Nitrate Propellant Emissions", in: "Environmental Aspects of Rocket and Gun Propulsion" AGARD Conference Proceedings 559, Neuilly sur Seine 1995, pp. 16.1-16.12.

(8) J. H. Kim, "Preparation of Phase Stabilized Ammonium Nitrate (PSAN) by a Salting Out Process", J. Chem. Eng. Japan 30(2), 336-338, 1997.

(9) B. S. Park, K. B. Lee, and C. S. Choi, "Evaluation of the Phase Stabilized Ammonium Nitrate Recrystallized from the Ternary System $\mathrm{NH}_{4} \mathrm{NO}_{3}-\mathrm{KNO}_{3}-\mathrm{H}_{2} \mathrm{O}$ ", 24th Int. Annual Conference of ICT, Karlsruhe, Germany, June 29-July 2, 1993, pp. 4.1-4.14.

(10) V. A. Koroban, Y. N. Burtsev, F. R. Alimov, A. D. Haustov, V. A. Dubovik, and V. A. Teselkin, "Thermal Decomposition Features of Ammonium Nitrate and its Boron Mixture under High Pressures", Propellants, Explosives, Pyrotechnics 19, 307 $310,1994$.

(11) N. Koga and H. Tanaka, "Part 3: Non-Isothermal Mass-Loss Process of Molten $\mathrm{NH}_{4} \mathrm{NO}_{3}$ ", Thermochimica Acta 240, 141$151,1994$.

(12) N. Koga and H. Tanaka, "Effect of Sample Mass on the Kinetics of Thermal Decomposition of a Solid, Part 1: Isothermal MassLoss Process of Molten $\mathrm{NH}_{4} \mathrm{NO}_{3}$ ", Thermochimica Acta 209, 127-134, 1992.

(13) T. B. Brill, P. J. Brush, and D. G. Patil, "Thermal Decomposition of Energetic Materials 58. Chemistry of Ammoniun Nitrate and Ammoniun Dinitramide Near the Burning Surface Temperature", Combustion and Flame 92, 178-186, 1993.

(14) D. G. Patil, S. R. Jain, and T. B. Brill, "Thermal Decomposition of Energetic Materials 55. On the Fast Thermolysis Mechanism of Ammoniun Nitrate and its Mixtures with Magnesium and Carbon", Propellants, Explosives, Pyrotechnics 17, 99-105, 1992. 
(15) K. R. Brower, J. C. Oxley, and M. Tewari, "Evidence for Homolytic Decomposition of Ammonium Nitrate at High Temperature", J. Phys. Chem. 93, 4029-4033, 1989.

(16) T. P. Russell and T. B. Brill, "Thermal Decomposition of Energetic Materials 31. Fast Thermolysis of Ammoniun Nitrate, Ethylenediammonium Dinitrate and Hydrazinium Nitrate and the Relationship to the Burning Rate", Combustion and Flame 76, 393-401, 1989.

(17) W. A. Rosser, S. H. Inami, and H. Wise, "The Kinetics of Decomposition of Liquid Ammonium Nitrate", J. Phys. Chem. 67, 1753-1757, 1963.

(18) P. N. Simões, L. M. Pedroso, A. A. Portugal, and J. L. Campos, "Study of the Decomposition of Phase Stabilized Ammonium
Nitrate (PSAN) by Simultaneous Thermal Analysis: Determination of Kinetic Parameters", Thermochimica Acta 319, 55-65, 1998.

(19) P. N. Simões, L. M. Pedroso, A. A. Portugal, and J. L. Campos, "Study of the Thermal Decomposition of Solid Mixtures of 2-Oxy-4,6-Dinitramine-s-Triazine (DNAM) and Phase Stabilized Ammonium Nitrate (PSAN)", Thermochimica Acta 364, 71-85, 2000.

(20) M. A. Meyers, "Dynamic Behavior of Materials", Wiley, New York, 1984, chapt. 5-7.

(Received December 16, 1999; Ms 1999/80) 